\title{
EQUIVALENCE CLASSES OF EXACT MODULE CATEGORIES OVER GRADED TENSOR CATEGORIES
}

\author{
ADRIANA MEJÍA CASTAÑO AND MARTÍN MOMBELLI
}

\begin{abstract}
We describe equivalence classes of exact indecomposable module categories over a finite graded tensor category. When applied to a pointed fusion category, our results coincide with the ones obtained in [11].
\end{abstract}

\section{INTRODUCTION}

Let $\mathcal{C}$ be a finite tensor category. A $\mathcal{C}$-module category consists of an abelian category $\mathcal{M}$ equipped with an action functor $\mathcal{C} \times \mathcal{M} \rightarrow \mathcal{M}$, satisfying certain associativity and unit axioms. The theory of representations of tensor categories has proven to be a powerful tool. In [5], the authors introduce the notion of exact module category, and as an intereseting problem, the classification of indecomposable exact module categories over a fixed finite tensor category.

Let $G$ be a finite group, and $\mathcal{D}=\oplus_{g \in G} \mathcal{C}_{g}$ be a $G$-graded tensor category. This family of tensor categories has been studied in [4]. In [10] and [7] the authors classify semisimple indecomposable modules over a semisimple $G$ graded tensor category $\mathcal{D}$ in terms of semisimple indecomposable modules over $\mathcal{C}_{1}$ and certain cohomological data. This paper is devoted to explain this classification, in the non-semisimple setting, using a different approach, inspired on results of [10, Section 8]. Our classification, when applied to a pointed fusion category, recovers the results obtained in [11]. Although very few results in this paper are new, we believe that the presentation of the results is our main contribution. We tried to be as self-contained as possible.

The contents of the paper are the following. In Section 2 we give an account of all the necessary preliminaries on finite tensor categories and their representations. We recall the notion of internal Hom as an important tool in the study of module categories. In Section 3 we recall the definition of graded tensor category, and some results concerning the restriction and induction of module categories. In Section 4 we start with the classification of exact indecomposable module categories over a fixed $G$-graded tensor category $\mathcal{D}=\oplus_{g \in G} \mathcal{C}_{g}$.

Date: March 22, 2018. 
We aim to recover results from [10, Section 8]. We show that indecomposable exact module categories over $\mathcal{D}=\oplus_{g \in G} \mathcal{C}_{g}$ are parametrized by collections $\left(H,\left\{A_{g}\right\}_{g \in H}, \beta\right)$ where

- $H \subseteq G$ is a subgroup;

- $A_{1}=A$ is an algebra in $\mathcal{C}=\mathcal{C}_{1}$ such that $\mathcal{C}_{A}$ is exact indecomposable;

- $A_{h}$ is an invertible $A$-bimodule in $\mathcal{C}_{h}$, for any $h \in H$;

- for any $f, h \in H$ there are bimodule isomorphisms $A_{f} \otimes_{A} A_{h} \simeq A_{f h}$;

- $\beta: H \times H \rightarrow \mathbb{k}^{\times}$is a normalized 2-cochain such that $1=d \beta \omega^{A}$.

Here $\omega^{A}$ is a 3 -cocycle for $H$ associated to the module category $\mathcal{C}_{A}$. We also prove that two collections $\left(H,\left\{A_{g}\right\}_{g \in H}, \beta\right),\left(F,\left\{B_{f}\right\}_{f \in F}, \gamma\right)$ give equivalent module categories if and only if there exists $g \in G$, and an invertible $(B, A)$ bimodule $C \in{ }_{B}\left(\mathcal{C}_{g}\right)_{A}$ such that

- $F=g g^{-1}$;

- $B_{g h g^{-1}} \otimes_{B} C \simeq C \otimes_{A} A_{h}$, for all $h \in H$;

- the class of $\beta^{-1} \Omega_{g}^{A} \gamma^{g}$ is trivial in $H^{2}\left(H, \mathbb{k}^{\times}\right)$.

Here $\Omega_{g}^{A}: G \times G \rightarrow \mathbb{k}^{\times}$is a certain 2-cochain associated to the module category $\mathcal{C}_{A}$. The 2 -cochain $\Omega_{g}^{A}$ does not appear in the results of $[10$, Section 6 ] when the authors study equivalence classes of certain cohomological data classifying module categories.

When $\mathcal{D}$ is a pointed fusion category, the classification coincide with the results obtained in [11, Theorem 1.1]. This is explained in Section 4.3.

Finally, we briefly explain results from [10, Section 8], reconciling our approach with the results presented in [10, Theorem 2].

1.1. Preliminaries and Notation. We shall work over an algebraically closed field $\mathbb{k}$ of characteristic 0 . All vector spaces are assumed to be over $\mathbb{k}$. If $\mathcal{C}, \mathcal{D}, \mathcal{A}, \mathcal{E}$ are categories, $F, G: \mathcal{C} \rightarrow \mathcal{D}, H: \mathcal{D} \rightarrow \mathcal{A}, J: \mathcal{E} \rightarrow \mathcal{C}$ are functors, and $\eta: F \rightarrow G$ is a natural transformation, we shall denote by $H \eta: H \circ F \rightarrow H \circ G$, and $\eta J: F \circ J \rightarrow G \circ J$, the natural transformations defined by

$$
(H \eta)_{X}=H\left(\eta_{X}\right), \quad(\eta J)_{Y}=\eta_{J(Y)}, \text { for any } X \in \mathcal{C}, Y \in \mathcal{E} .
$$

\section{RePRESENTATIONS OF TENSOR CATEGORIES}

For basic notions on finite tensor categories we refer to [3], [5]. Let $\mathcal{C}$ be a finite tensor category over $\mathbb{k}$. A (left) module over $\mathcal{C}$ is a finite $\mathbb{k}$-linear abelian category $\mathcal{M}$ together with a $\mathbb{k}$-bilinear bifunctor $\bar{\otimes}: \mathcal{C} \times \mathcal{M} \rightarrow \mathcal{M}$, exact in each variable, endowed with natural associativity and unit isomorphisms

$$
m_{X, Y, M}:(X \otimes Y) \bar{\otimes} M \rightarrow X \bar{\otimes}(Y \bar{\otimes} M), \quad \ell_{M}: \mathbf{1} \bar{\otimes} M \rightarrow M .
$$

These isomorphisms are subject to the following conditions:

$$
\begin{gathered}
m_{X, Y, Z \bar{\otimes} M} m_{X \otimes Y, Z, M}=\left(\operatorname{id}_{X} \otimes m_{Y, Z, M}\right) m_{X, Y \otimes Z, M}\left(\alpha_{X, Y, Z} \bar{\otimes} \operatorname{id}_{M}\right), \\
\left(\operatorname{id}_{X} \bar{\otimes} l_{M}\right) m_{X, \mathbf{1}, M}=\operatorname{id}_{X \bar{\otimes} M},
\end{gathered}
$$


for any $X, Y, Z \in \mathcal{C}, M \in \mathcal{M}$. Here $\alpha$ is the associativity constraint of $\mathcal{C}$. Sometimes we shall also say that $\mathcal{M}$ is a $\mathcal{C}$-module or a $\mathcal{C}$-module category. In a similar way, one can define right modules and bimodules. See for example [9].

Let $\mathcal{M}$ and $\mathcal{M}^{\prime}$ be a pair of $\mathcal{C}$-modules. We say that a functor $F: \mathcal{M} \rightarrow$ $\mathcal{M}^{\prime}$ is a module functor if it is equipped with natural isomorphisms

$$
c_{X, M}: F(X \bar{\otimes} M) \rightarrow X \bar{\otimes} F(M),
$$

$X \in \mathcal{C}, M \in \mathcal{M}$, such that for any $X, Y \in \mathcal{C}, M \in \mathcal{M}$ :

$$
\begin{aligned}
\left(\operatorname{id}_{X} \bar{\otimes} c_{Y, M}\right) c_{X, Y \bar{\otimes} M} F\left(m_{X, Y, M}\right) & =m_{X, Y, F(M)} c_{X \otimes Y, M} \\
\ell_{F(M)} c_{\mathbf{1}, M} & =F\left(\ell_{M}\right) .
\end{aligned}
$$

There is a composition of module functors: if $\mathcal{M}^{\prime \prime}$ is another $\mathcal{C}$-module and $(G, d): \mathcal{M}^{\prime} \rightarrow \mathcal{M}^{\prime \prime}$ is another module functor then the composition

$$
(G \circ F, e): \mathcal{M} \rightarrow \mathcal{M}^{\prime \prime}, \quad e_{X, M}=d_{X, F(M)} \circ G\left(c_{X, M}\right),
$$

is also a module functor.

We denote by $\operatorname{Fun}_{\mathcal{C}}\left(\mathcal{M}, \mathcal{M}^{\prime}\right)$ the category whose objects are module functors $(F, c)$ from $\mathcal{M}$ to $\mathcal{M}^{\prime}$. A morphism between module functors $(F, c)$ and $(G, d) \in \operatorname{Fun}_{\mathcal{C}}\left(\mathcal{M}, \mathcal{M}^{\prime}\right)$ is a natural module transformation, that is, a natural transformation $\alpha: F \rightarrow G$ such that for any $X \in \mathcal{C}, M \in \mathcal{M}$ :

$$
d_{X, M} \alpha_{X \bar{\otimes} M}=\left(\operatorname{id}_{X} \bar{\otimes} \alpha_{M}\right) c_{X, M} .
$$

Two module functors $F, G$ are equivalent if there exists a natural module isomorphism $\alpha: F \rightarrow G$.

Two $\mathcal{C}$-modules $\mathcal{M}$ and $\mathcal{M}^{\prime}$ are equivalent if there exist module functors $F: \mathcal{M} \rightarrow \mathcal{M}^{\prime}, G: \mathcal{M}^{\prime} \rightarrow \mathcal{M}$, and natural module isomorphisms $\operatorname{Id}_{\mathcal{M}} \rightarrow$ $F \circ G, \operatorname{Id}_{\mathcal{M}^{\prime}} \rightarrow G \circ F$.

A module is indecomposable if it is not equivalent to a direct sum of two non trivial modules. Recall from [5], that a module $\mathcal{M}$ is exact if $\mathcal{M}$ for any projective object $P \in \mathcal{C}$ the object $P \bar{\otimes} M$ is projective in $\mathcal{M}$, for all $M \in \mathcal{M}$. Right module categories and bimodule categories are defined similarly. See for example [9]. If $\mathcal{M}, \mathcal{N}$ are $\mathcal{C}$-bimodule categories, we denote by $\mathcal{M} \nabla_{\mathcal{C}} \mathcal{N}$ the balanced tensor product over $\mathcal{C}$. See [8].

The next result seems to be well-known.

Lemma 2.1. Let $\mathcal{M}$ be a $\mathcal{C}$-module category. If $X \in \mathcal{C}, M \in \mathcal{M}$ are non zero objects, then $X \bar{\otimes} M \neq 0$.

Proof. Let us assume that $X \bar{\otimes} M=0$. The map

$$
M \stackrel{l_{M}}{\longrightarrow} \mathbf{1} \bar{\otimes} M \stackrel{\operatorname{coev}_{X} \bar{\otimes} \operatorname{id}_{M}}{\longrightarrow}\left({ }^{*} X \otimes X\right) \bar{\otimes} M \stackrel{m^{*} X, X, M}{\longrightarrow}{ }^{*} X \bar{\otimes}(X \bar{\otimes} M)=0,
$$

is the zero morphism. Since the coevaluation $\operatorname{coev}_{X}$ is a monomorphism, and $\bar{\otimes}$ is bi-exact, then $\operatorname{coev}_{X} \bar{\otimes} \mathrm{id}{ }_{M}$ is a monomorphism. Thus, the above composition is also a monomorphism. Hence $M=0$. 
2.1. The internal Hom. Let $\mathcal{C}$ be a finite tensor category and $\mathcal{M}$ be a $\mathcal{C}$ module. For any pair of objects $N, M \in \mathcal{M}$, the internal Hom is an object $\underline{\operatorname{Hom}}_{\mathcal{C}}(N, M) \in \mathcal{C}$ representing the functor $\operatorname{Hom}_{\mathcal{M}}(-\bar{\otimes} N, M): \mathcal{C} \rightarrow$ vect $_{\mathbb{k}}$. That is, there are natural isomorphisms

$$
\operatorname{Hom}_{\mathcal{C}}\left(X, \underline{\operatorname{Hom}}_{\mathcal{C}}(N, M)\right) \simeq \operatorname{Hom}_{\mathcal{M}}(X \bar{\otimes} N, M) \text {, for all } X \in \mathcal{C} .
$$

Proposition 2.2. [5, Thm. 3.17] For each object $0 \neq M \in \mathcal{M}$ the internal Hom $A=\underline{\operatorname{Hom}}_{\mathcal{C}}(M, M)$ is an algebra in $\mathcal{C}$. If $N$ is a subobject of $M$ then $\underline{\operatorname{Hom}}_{\mathcal{C}}(M, N)$ is a right ideal of $A$. Moreover $\underline{\operatorname{Hom}}_{\mathcal{C}}(M,-): \mathcal{M} \rightarrow \mathcal{C}_{A}$ is a $\mathcal{C}$-module functor. If $\mathcal{M}$ is exact indecomposable, the functor

$$
\underline{\operatorname{Hom}}_{\mathcal{C}}(M,-): \mathcal{M} \rightarrow \mathcal{C}_{A}
$$

is an equivalence of $\mathcal{C}$-module categories.

Using the above Proposition, when dealing with exact indecomposable module categories, we can restricts ourself only with those of the form $\mathcal{C}_{A}$, for some algebra $A \in \mathcal{C}$. The next result was given in [5].

Proposition 2.3. Let $A, B \in \mathcal{C}$ algebras such that the module categories $\mathcal{C}_{A}, \mathcal{C}_{B}$ are exact indecomposable. There exists an equivalence of categories

$$
\operatorname{Fun}_{\mathcal{C}}\left(\mathcal{C}_{A}, \mathcal{C}_{B}\right) \simeq{ }_{A} \mathcal{C}_{B}^{\text {op }}
$$

\section{Graded tensor CATEgories}

An important family of examples of tensor categories come from group extensions. Given a finite group $G$, a (faithful) $G$-grading on a finite tensor category $\mathcal{D}$ is a decomposition $\mathcal{D}=\oplus_{g \in G} \mathcal{C}_{g}$, where $\mathcal{C}_{g}$ are non-zero full abelian subcategories of $\mathcal{D}$ such that

$$
\otimes: \mathcal{C}_{g} \times \mathcal{C}_{h} \rightarrow \mathcal{C}_{g h}, \text { for all } g, h \in G .
$$

In this case, we say that $\mathcal{D}$ is a $G$-extension of $\mathcal{C}:=\mathcal{C}_{1}$. These extensions were studied and classified in [4] in terms of the Brauer-Picard group of the category $\mathcal{C}$ and certain cohomological data.

Example 3.1. Let $G$ be a finite group and $\omega \in H^{3}\left(G, \mathbb{k}^{\times}\right) 3$-cocycle. The category $\mathcal{C}(G, \omega)$ has objects finite dimensional $G$-graded vector spaces, with associativity constraint defined by

$$
a_{X, Y, Z}((x \otimes y) \otimes z)=\omega(g, h, f) x \otimes(y \otimes z),
$$

for any $X, Y, Z \in \mathcal{C}(G, \omega)$, and any homogeneous elements $x \in X_{g}, y \in$ $Y_{h}, z \in Z_{f}$. The tensor category $\mathcal{C}(G, \omega)$ is an example of a $G$-extension of the category of finite dimensional vector spaces vect $\mathbb{k}_{\mathbb{k}}$. More precisely, $\mathcal{C}(G, \omega)=\oplus_{g \in G}$ vect $_{g}$, where vect $g$ denotes the category of finite dimensional vector spaces supported in the component $g$.

We list some important properties of these categories. 
Proposition 3.2. Assume $\mathcal{D}=\oplus_{g \in G} \mathcal{C}_{g}$ is a $G$-graded extension of $\mathcal{C}$. The following statements hold.

1. For any $g, h \in G$, the tensor product of $\mathcal{D}$ induces an equivalence of $\mathcal{C}$-bimodule categories

$$
M_{g, h}: \mathcal{C}_{g} \otimes_{\mathcal{C}} \mathcal{C}_{h} \rightarrow \mathcal{C}_{g h}, \quad M_{g, h}(X \otimes Y)=X \otimes Y .
$$

2. The associativity constraints of $\mathcal{D}$ induce natural $\mathcal{C}$-bimodule isomorphisms

$$
\alpha_{f, g, h}: M_{f g, h}\left(M_{f, g} \otimes_{\mathcal{C}} \operatorname{Id}_{\mathcal{C}_{h}}\right) \rightarrow M_{f, g h}\left(\operatorname{Id}_{\mathcal{C}_{f}} \otimes_{\mathcal{C}} M_{g, h}\right),
$$

for any $f, g, h \in G$, such that

$$
\begin{gathered}
M_{f, g h k}\left(\operatorname{Id}_{\mathcal{C}_{f}} \bigotimes_{\mathcal{C}} \alpha_{g, h, k}\right) \circ \alpha_{f, g h, k}\left(\operatorname{Id}_{\mathcal{C}_{f}} \nabla_{\mathcal{C}} M_{g, h} \otimes_{\mathcal{C}} \operatorname{Id}_{\mathcal{C}_{k}}\right) \circ M_{f g h, k}\left(\alpha_{f, g, h} \bigotimes_{\mathcal{C}} \operatorname{Id}_{\mathcal{C}_{k}}\right) \\
=\alpha_{f, g, h k}\left(\operatorname{Id}_{\mathcal{C}_{f}} \nabla_{\mathcal{C}} \operatorname{Id}_{\mathcal{C}_{g}} \nabla_{\mathcal{C}} M_{h, k}\right) \circ \alpha_{f g, h, k}\left(M_{f, g} \otimes_{\mathcal{C}} \operatorname{Id}_{\mathcal{C}_{h}} \nabla_{\mathcal{C}} \operatorname{Id}_{\mathcal{C}_{k}}\right) .
\end{gathered}
$$

Proof. It follows, mutatis mutandis, from the proof of [4, Theorem 6.1], in the non-semisimple case. See also Equation (51) of [4].

For any $f, g \in G$, we can choose $\mathcal{C}$-bimodule equivalences

$$
\bar{M}_{f, g}: \mathcal{C}_{f g} \rightarrow \mathcal{C}_{f} \otimes_{\mathcal{C}} \mathcal{C}_{g}
$$

such that $M_{f, g} \circ \bar{M}_{f, g}=\operatorname{Id}_{\mathcal{C}_{f g}}$.

If $\widetilde{\mathcal{D}}=\oplus_{g \in G} \widetilde{\mathcal{C}}_{g}$ is another $G$-graded tensor category, a graded tensor functor $(F, \xi): \mathcal{D} \rightarrow \widetilde{\mathcal{D}}$ is a tensor functor such that $F\left(\mathcal{C}_{g}\right) \subseteq \widetilde{\mathcal{C}}_{g}$, for any $g \in$ $G$. This means that there are natural isomorphisms $\xi_{X, Y}: F(X) \otimes F(Y) \rightarrow$ $F(X \otimes Y)$ such that

$$
\xi_{X, Y \otimes Z}\left(\operatorname{id}_{F(X)} \otimes \xi_{Y, Z}\right) \widetilde{\alpha}_{F(X), F(Y), F(Z)}=F\left(\alpha_{X, Y, Z}\right) \xi_{X \otimes Y, Z}\left(\xi_{X, Y} \otimes \operatorname{id}_{F(Z)}\right) .
$$

Here $\alpha, \widetilde{\alpha}$ denote the associativity constraint of the tensor categories $\mathcal{D}, \widetilde{\mathcal{D}}$. If we denote by $F_{g}: \mathcal{C}_{g} \rightarrow \widetilde{\mathcal{C}}_{g}$ the restriction of the functor $F$ and by $N_{f, g}: \widetilde{\mathcal{C}}_{f} \otimes \widetilde{\mathcal{C}}_{g} \rightarrow \widetilde{\mathcal{C}}_{f g}$ the restriction of the tensor product of $\widetilde{\mathcal{D}}$, then the tensoriality of the functor $F$ implies that there are natural isomorphisms $\xi_{f, g}: N_{f, g}\left(F_{f} \otimes F_{g}\right) \rightarrow F_{f g} M_{f, g}$ (these are the restrictions of the natural isomorphisms $\xi$ to the category $\mathcal{C}_{f} \times \mathcal{C}_{g}$ ), and equation (3.3) implies that

$$
\begin{aligned}
\left(\xi_{f g, h} \circ \mathrm{id}_{\operatorname{Id}_{f} \otimes M_{g, h}}\right)\left(\operatorname{id}_{N_{f, g h}} \circ\left(\operatorname{id}_{f} \otimes \xi_{g, h}\right)\right) \widetilde{\alpha}_{f, g, h}\left(F_{f} \otimes F_{g} \otimes F_{h}\right)= \\
=\left(F_{f g h} \alpha_{f, g, h}\right)\left(\xi_{f g, h} \circ \mathrm{id}_{M_{f, g} \otimes \operatorname{Id}_{h}}\right)\left(\operatorname{id}_{N_{f g, h}} \circ\left(\xi_{f, g} \otimes \operatorname{id}_{h}\right)\right) .
\end{aligned}
$$

Here, for any $g \in G$, we denote $\operatorname{Id}_{g}: \mathcal{C}_{g} \rightarrow \mathcal{C}_{g}$ the identity functor, and $\operatorname{id}_{g}: F_{g} \rightarrow F_{g}$ the identity natural transformation.

Remark 3.3. For any $G$-graded tensor category $\mathcal{D}$ there exists a skeletal $G$ graded tensor category $\widetilde{\mathcal{D}}$ and a graded tensor equivalence $(F, \xi): \mathcal{D} \rightarrow \widetilde{\mathcal{D}}$. Hence, we can always work over a skeletal graded tensor category. 
3.1. Induction and restriction of module categories. Let $\mathcal{D}$ be a tensor category, and let $\mathcal{C}$ be a tensor subcategory of $\mathcal{D}$. Assume that $\mathcal{N}$ is a $\mathcal{C}$-module. We shall denote by $\operatorname{Ind}_{\mathcal{C}}^{\mathcal{D}}(\mathcal{N}):=\mathcal{D} \otimes_{\mathcal{C}} \mathcal{N}$ the induced $\mathcal{D}$-module, where the $\mathcal{D}$-action is induced by the tensor product of $\mathcal{D}[6$, Proposition 2.13]. Let $\mathcal{M}$ be a $\mathcal{D}$-module, we shall denote by $\operatorname{Res}_{\mathcal{C}}^{\mathcal{D}} \mathcal{M}$ the restricted $\mathcal{C}$-module.

The following result seems to be well-known. We include the proof for completeness' sake. Part of it is contained in [10, Corollary 9], see also [6, Proposition 3.7].

Lemma 3.4. Assume that we have a decomposition $\mathcal{D}=\mathcal{C} \oplus \mathcal{C}^{\prime}$ as abelian categories, such that $\mathcal{C}$ is a tensor subcategory. Let $\mathcal{M}$ be an exact indecomposable $\mathcal{D}$-module category such that it decomposes as $\mathcal{M}=\oplus_{i=1}^{n} \mathcal{M}_{i}$, where $\mathcal{M}_{i}$ are exact indecomposable $\mathcal{C}$-modules. Assume also that every time we choose non zero objects $X \in \mathcal{C}^{\prime}, N \in \mathcal{M}_{1}$, then $X \bar{\otimes} N \notin \mathcal{M}_{1}$. Take $0 \neq M \in \mathcal{M}_{1}$, and $A=\underline{\operatorname{Hom}}_{\mathcal{D}}(M, M)$. Then $A \in \mathcal{C}, \mathcal{M}_{1} \simeq \mathcal{C}_{A}$, and there is an equivalence of $\mathcal{D}$-modules

$$
\mathcal{M} \simeq \operatorname{Ind}_{\mathcal{C}}^{\mathcal{D}}\left(\mathcal{C}_{A}\right)
$$

Proof. Take arbitrary $V \in \mathcal{C}^{\prime}$. Then by $(2.7)$

$$
\operatorname{Hom}_{\mathcal{D}}(V, A) \simeq \operatorname{Hom}_{\mathcal{M}}(V \bar{\otimes} M, M) .
$$

Since $V \bar{\otimes} M \notin \mathcal{M}_{1}$, then $\operatorname{Hom}_{\mathcal{D}}(V, A)=0$, and $A \in \mathcal{C}$. If $X \in \mathcal{C}$, there are isomorphisms

$$
\begin{aligned}
& \operatorname{Hom}_{\mathcal{C}}\left(X, \underline{\operatorname{Hom}}_{\mathcal{C}}(M, M)\right) \simeq \operatorname{Hom}_{\mathcal{M}_{1}}(X \bar{\otimes} M, M), \\
& \operatorname{Hom}_{\mathcal{D}}\left(X, \underline{\operatorname{Hom}}_{\mathcal{D}}(M, M)\right) \simeq \operatorname{Hom}_{\mathcal{M}}(X \bar{\otimes} M, M) .
\end{aligned}
$$

Since $\operatorname{Hom}_{\mathcal{M}_{1}}(X \bar{\otimes} M, M)=\operatorname{Hom}_{\mathcal{M}}(X \bar{\otimes} M, M)$, then

$$
\operatorname{Hom}_{\mathcal{C}}\left(X, \operatorname{Hom}_{\mathcal{C}}(M, M)\right) \simeq \operatorname{Hom}_{\mathcal{D}}(X, A) \simeq \operatorname{Hom}_{\mathcal{C}}(X, A) .
$$

Whence, $A=\underline{\operatorname{Hom}}_{\mathcal{C}}(M, M)$, and $\mathcal{M}_{1} \simeq \mathcal{C}_{A}$ as $\mathcal{C}$-modules. Since $\mathcal{M}$ is indecomposable, the action $\mathcal{D} \nabla_{\mathcal{C}} \mathcal{M}_{1} \rightarrow \mathcal{M}, X \otimes V \mapsto X \bar{\otimes} V$ is an equivalence of $\mathcal{D}$-modules.

In the following Lemma we include some properties of the induced and restricted modules categories.

Lemma 3.5. Assume $\mathcal{D}=\oplus_{g \in G} \mathcal{C}_{g}$ is a $G$-graded extension of $\mathcal{C}=\mathcal{C}_{1}$. Let $\mathcal{N}$ be a $\mathcal{C}$-module and $\mathcal{M}$ a $\mathcal{D}$-module. The following statements hold.

1. $\mathcal{M}$ is an exact $\mathcal{D}$-module, if and only if, $\operatorname{Res}_{\mathcal{C}}^{\mathcal{D}} \mathcal{M}$ is an exact $\mathcal{C}$ module.

2. If $\mathcal{N}$ is exact (indecomposable) then $\operatorname{Ind}_{\mathcal{C}}^{\mathcal{D}}(\mathcal{N})$ is exact (indecomposable).

Proof. 1. Since $\mathcal{C} \subset \mathcal{D}$ is a tensor subcategory, then it follows from $[2$, Corollary 2.5], that if $\operatorname{Res}_{\mathcal{C}}^{\mathcal{D}} \mathcal{M}$ is exact $\mathcal{C}$-module, then $\mathcal{M}$ is exact as a $\mathcal{D}$ module. Now, assume that $\mathcal{M}$ is exact. Let be $P \in \mathcal{C}$ a projective object 
and $X \in \operatorname{Res}{ }_{\mathcal{C}}^{\mathcal{D}} \mathcal{M}$. Since $P \in \mathcal{D}$ is projective, then $P \bar{\otimes} X$ is projective in $\operatorname{Res}_{\mathcal{C}}^{\mathcal{D}} \mathcal{M}$, hence $\operatorname{Res}_{\mathcal{C}}^{\mathcal{D}} \mathcal{M}$ is exact.

2. To prove the exactness of $\operatorname{Ind}_{\mathcal{C}}^{\mathcal{D}} \mathcal{N}$ we follow the argument of $[1$, Prop. 2.10]. Since $\operatorname{Ind}_{\mathcal{C}}^{\mathcal{D}} \mathcal{N}=\oplus_{g \in G} \mathcal{C}_{g} \otimes_{\mathcal{C}} \mathcal{N}$, then $\operatorname{Ind}_{\mathcal{C}}^{\mathcal{D}} \mathcal{N}$ is an exact $\mathcal{D}$-module if and only if $\mathcal{C}_{g} \nabla_{\mathcal{C}} \mathcal{N}$ is an exact $\mathcal{C}$-module category for any $g \in G$. It follows from [5, Lemma 3.30] that $\mathcal{C}_{g} \otimes_{\mathcal{C}} \mathcal{N}$ is an exact $\operatorname{End}_{\mathcal{C}}\left(\mathcal{C}_{g}\right)$-module category. Using [4, Prop. 4.2], since $\mathcal{C}_{g}$ is an invertible $\mathcal{C}$-bimodule category, $\operatorname{End}_{\mathcal{C}}\left(\mathcal{C}_{g}\right) \simeq \mathcal{C}$. Hence $\mathcal{C}_{g} \nabla_{\mathcal{C}} \mathcal{N}$ is an exact $\mathcal{C}$-module category.

Assume that $\mathcal{N}$ is an indecomposable $\mathcal{C}$-module category, and we can decompose $\operatorname{Ind}_{\mathcal{C}}^{\mathcal{D}} \mathcal{N}=\mathcal{M}_{1} \oplus \mathcal{M}_{2}$ as $\mathcal{D}$-modules. By restriction $\mathcal{N} \simeq \mathbf{1}_{\mathcal{C}} \mathcal{N}=$ $\mathcal{N}_{1} \oplus \mathcal{N}_{2}$ as $\mathcal{C}$-modules, then $\mathcal{N}_{1}=0$ or $\mathcal{N}_{2}=0$. Suppose $\mathcal{N}_{2}=0$, thus $\mathcal{N} \subset \mathcal{M}_{1}$. Take a non-zero object $0 \neq M \in \mathcal{M}_{2}$. We can assume that $M \in \mathcal{C}_{g} \bigotimes_{\mathcal{C}} \mathcal{N}$, for some $g \in G$. Take $0 \neq Y \in \mathcal{C}_{g^{-1}}$, then, by Lemma 2.1 $0 \neq Y \bar{\otimes} M \in \mathcal{N}$ is a non-zero object. Since the restriction of the tensor product maps $\mathcal{C}_{g^{-1}} \times \mathcal{C}_{g} \rightarrow \mathcal{C}$, then $Y \bar{\otimes} M \in \mathcal{N}_{2}$, which contradicts our assumption.

\section{Module CATEgories over $G$-Graded tensor CATEgories}

Let $G$ be a finite group and $\mathcal{D}=\oplus_{g \in G} \mathcal{C}_{g}$ be a $G$-graded extension of $\mathcal{C}=\mathcal{C}_{1}$. In [10], [6], [7] the authors, independently, classify semisimple indecomposable modules over $\mathcal{D}$ in terms of exact $\mathcal{C}$-modules and certain cohomological data. We shall recall these results dropping the semisimplicity condition and using instead the exactness hypothesis.

We shall assume that $\mathcal{D}$ is a skeletal graded tensor category. See remark 3.3 .

For any subset $F \subset G$, we define $\mathcal{C}_{F}=\oplus_{g \in F} \mathcal{C}_{g}$. In case $F$ is a subgroup, $\mathcal{C}_{F}$ is a tensor subcategory of $\mathcal{D}$. If $\mathcal{N}$ is a $\mathcal{C}$-module, we say that $\mathcal{N}$ is $F$-equivariant if $\mathcal{C}_{f} \square_{\mathcal{C}} \mathcal{N} \simeq \mathcal{N}$ as left $\mathcal{C}$-module categories, for any $f \in F$.

Lemma 4.1. Assume $A \in \mathcal{C}$ be an algebra such that $\mathcal{C}_{A}$ is an exact indecomposable $\mathcal{C}$-module category. The following holds:

1. For any $g \in G, \mathcal{C}_{g} \otimes_{\mathcal{C}} \mathcal{C}_{A}$ is an exact indecomposable $\mathcal{C}$-module.

2. The tensor product of $\mathcal{D}$ induces an equivalence of left $\mathcal{D}$-module categories $\left(\mathcal{C}_{g}\right)_{A} \simeq \mathcal{C}_{g} \nabla_{\mathcal{C}} \mathcal{C}_{A}$.

3. The natural module isomorphism

$$
\begin{gathered}
M_{f g, h}\left(M_{f, g} \otimes_{\mathcal{C}} \operatorname{Id}_{\mathcal{C}_{h}}\right) \otimes \operatorname{Id}_{\mathcal{C}_{A}} \stackrel{\alpha_{f, g, h} \otimes \mathrm{id}}{\longrightarrow} M_{f, g h}\left(\operatorname{Id}_{\mathcal{C}_{f}} \otimes_{\mathcal{C}} M_{g, h}\right) \otimes \operatorname{Id}_{\mathcal{C}_{A}}, \\
\text { are determined by scalars } \omega_{f, g, h}^{A} \in \mathbb{k}^{\times} \text {, for any } f, g, h \in G .
\end{gathered}
$$

4. $\omega^{A} \in Z^{3}\left(G, \mathbb{k}^{\times}\right)$is a 3-cocycle. Its cohomology class does not change if we change the monoidal equivalence class of $\mathcal{D}$. If $\mathcal{C}_{B} \simeq \mathcal{C}_{A}$ are equivalent as $\mathcal{C}$-modules, then $\omega^{A}=\omega^{B}$. 
Proof. 1. This statement was proved in (the proof of) Lemma 3.5 (2).

2. The proof follows, mutatis mutandis, from the proof of [10, Lemma 24] in the non-semisimple case.

3. For any finite tensor category $\mathcal{D}$, and any exact indecomposable $\mathcal{D}$ module $\mathcal{M}$, the identity functor $\operatorname{Id}_{\mathcal{M}}$ is a simple object in $\operatorname{End}_{\mathcal{D}}(\mathcal{M})$. Since the $\mathcal{C}$-module category $\mathcal{C}_{f} \nabla_{\mathcal{C}} \mathcal{C}_{g} \nabla_{\mathcal{C}} \mathcal{C}_{h} \nabla_{\mathcal{C}} \mathcal{C}_{A}$ is equivalent to $\left(\mathcal{C}_{f g h}\right)_{A}$, then there is an equivalence of categories

$$
\operatorname{Fun}_{\mathcal{C}}\left(\left(\mathcal{C}_{f g h}\right)_{A},\left(\mathcal{C}_{f g h}\right)_{A}\right) \simeq{ }_{A}\left(\mathcal{C}_{f g h}\right)_{A}^{\text {op }}
$$

The later category being skeletal, since $\mathcal{D}$ is skeletal. The module functors $M_{f g, h}\left(M_{f, g} \nabla_{\mathcal{C}} \operatorname{Id}_{\mathcal{C}_{h}}\right) \otimes \operatorname{Id}_{\mathcal{C}_{A}}, M_{f, g h}\left(\operatorname{Id}_{\mathcal{C}_{f}} \nabla_{\mathcal{C}} M_{g, h}\right) \otimes \operatorname{Id}_{\mathcal{C}_{A}}$ correspond, under equivalence $(4.1)$, to invertible objects. Since $\left(\mathcal{C}_{f g h}\right)_{A}$ is indecomposable, the identity functor is simple, then any invertible object is simple. Thus, natural transformations $\alpha_{f, g, h} \otimes$ id correspond to $\omega_{f, g, h}^{A}$ id .

4. It follows from (3.1) that $\omega^{A}$ is a 3-cocycle and from (3.4) that the cohomology class of $\omega^{A}$ does not change if we replace $\widetilde{\mathcal{D}}$ by another skeletal graded tensor category monoidally equivalent to $\mathcal{D}$.

Assume that $\Phi: \mathcal{C}_{A} \rightarrow \mathcal{C}_{B}, \Psi: \mathcal{C}_{B} \rightarrow \mathcal{C}_{A}$ are $\mathcal{C}$-module equivalences, and let be $\eta: \Phi \circ \Psi \rightarrow \operatorname{Id}_{\mathcal{C}_{B}}$ a natural module isomorphism. For any $f, g, h \in G$, let us denote

$$
F_{f, g, h}=M_{f g, h}\left(M_{f, g} \otimes_{\mathcal{C}} \operatorname{Id}_{\mathcal{C}_{h}}\right) \text {, and } G_{f, g, h}=M_{f, g h}\left(\operatorname{Id}_{\mathcal{C}_{f}} \otimes_{\mathcal{C}} M_{g, h}\right) .
$$

The commutativity of diagram

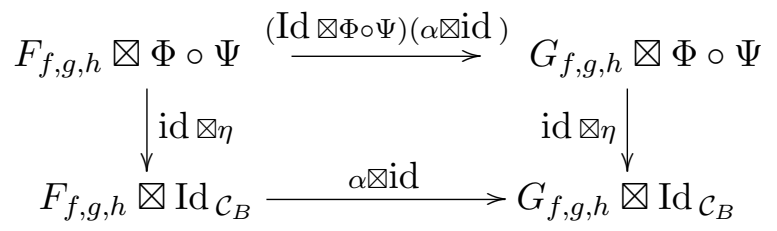

implies that $\omega^{A}=\omega^{B}$.

Remark 4.2. Let $G$ be a finite group, $\omega \in H^{3}\left(G, \mathbb{k}^{\times}\right)$be a 3 -cocycle, and $\mathcal{C}(G, \omega)$ be the fusion category described in Example 3.1. For any algebra $A \in \mathcal{C}(G, \omega)$ such that the module category $\mathcal{C}(G, \omega)_{A}$ is exact indecomposable, one can verify that $\omega^{A}=\omega$.

Definition 4.3. Assume $A \in \mathcal{C}$ be an algebra such that $\mathcal{C}_{A}$ is an exact indecomposable $\mathcal{C}$-module category. A type- $A$ datum for the module category $\mathcal{C}_{A}$ is a collection $\left(H,\left\{A_{h}\right\}_{h \in H}, \beta\right)$ where

- $H \subseteq G$ is a subgroup;

- $A_{1}=A$;

- $A_{h}$ is an invertible $A$-bimodule in $\mathcal{C}_{h}$, that is $A_{h} \in{ }_{A}\left(\mathcal{C}_{h}\right)_{A}$, for any $h \in H$;

- there are $A$-bimodule isomorphisms $A_{f} \otimes_{A} A_{h} \simeq A_{f h}$, for any $f, h \in$ $H$; 
- $\beta: H \times H \rightarrow \mathbb{k}^{\times}$is a 2-cochain such that for any $f, g, h \in H$

$$
\beta_{f, 1}=1=\beta_{1, f}, \quad \beta_{f, g} \beta_{f g, h}=\beta_{g, h} \beta_{f, g h} \omega_{f, g, h}^{A} .
$$

Let $B \in \mathcal{C}$ be another algebra, such that $\mathcal{C}_{B}$ is an exact indecomposable $\mathcal{C}$ module category. A type-A datum $\left(F,\left\{B_{f}\right\}_{f \in F}, \gamma\right)$ for the module category $\mathcal{C}_{B}$, is equivalent to the type-A datum $\left(H,\left\{A_{h}\right\}_{h \in H}, \beta\right)$ for $\mathcal{C}_{A}$ if

- $F=H$;

- there exists an invertible $(B, A)$-bimodule $C \in{ }_{B} \mathcal{C}_{A}$ together with $(B, A)$-bimodule isomorphisms

$$
B_{h} \otimes_{B} C \simeq C \otimes_{A} A_{h}, \text { for any } h \in H ;
$$

- there are scalars $\tau_{h} \in \mathbb{k}^{\times}$such that

$$
\tau_{l} \tau_{h} \tau_{h l}^{-1} \gamma_{h, l}=\beta_{h, l}, \text { for } l, h \in G .
$$

Remark 4.4. If $\left(H,\left\{A_{g}\right\}_{g \in H}, \beta\right)$ and $\left(H,\left\{B_{f}\right\}_{f \in F}, \gamma\right)$ are equivalent, then the functor $-\otimes_{B} C: \mathcal{C}_{B} \rightarrow \mathcal{C}_{A}$ is a $\mathcal{C}$-module equivalence, and using Lemma 4.1 (4), we get that the class of $\beta \gamma^{-1}$ is trivial in $H^{2}\left(H, \mathbb{k}^{\times}\right)$.

Remark 4.5. The idea of the definition of the type-A datum is taken from [10, Section 8].

Lemma 4.6. The existence of a type- $A$ datum $\left(H,\left\{A_{g}\right\}_{g \in H}, \beta\right)$ for a $\mathcal{C}$ module category $\mathcal{C}_{A}$ implies that $\mathcal{C}_{A}$ is $H$-equivariant.

Proof. Indeed, for any $h \in H$, the functors

$$
\Psi_{h}: \mathcal{C}_{h} \otimes_{\mathcal{C}} \mathcal{C}_{A} \rightarrow \mathcal{C}_{A}, \quad \Psi_{h}(X \otimes V)=(X \otimes V) \otimes_{A} A_{h^{-1}},
$$

are well-defined $\mathcal{C}$-module equivalences.

Now, we shall explain the classification of indecomposable exact $\mathcal{D}$-module categories obtained in [7] and [10]. This classification will be done in two steps. In the first step will associate to any indecomposable exact $\mathcal{D}$-module category a pair $(H, \mathcal{N})$, where $H \subseteq G$ is a subgroup, and $\mathcal{N}$ is an exact indecomposable $\mathcal{C}_{H}$-module category such that the restriction $\operatorname{Re}{ }_{C}^{\mathcal{C}_{H}} \mathcal{N}$ remains indecomposable as a $\mathcal{C}$-module. In the second step, using Proprosition 4.11, we shall associate, to any such pair $(H, \mathcal{N})$ a type-A datum for $\operatorname{Re} s_{\mathcal{C}}{ }^{\mathcal{C}_{H}} \mathcal{N}$.

4.1. First step. Let $H$ be a subgroup of $G$, and let $\mathcal{N}$ be an indecomposable exact $\mathcal{C}_{H}$-module category such that $\mathcal{M}=\operatorname{Res}_{\mathcal{C}}^{\mathcal{C}_{H}} \mathcal{N}$ is an exact indecomposable $\mathcal{C}$-module category. Under these assumptions, we shall call $(H, \mathcal{N})$ a type-1 pair.

Note that if $(H, \mathcal{N})$ is a type-1 pair, then, for any $g \in G$, the category $\mathcal{C}_{g H} \otimes_{\mathcal{C}_{H}} \mathcal{N}$ has an action of $\mathcal{C}_{g H g^{-1}}$, such that

$$
\operatorname{Res}_{\mathcal{C}}^{\mathcal{C}_{g g^{-1}}}\left(\mathcal{C}_{g H} \otimes_{\mathcal{C}_{H}} \mathcal{N}\right) \simeq \mathcal{C}_{g} \nabla_{\mathcal{C}} \mathcal{M}
$$

By Lemma 4.1 (1) $\mathcal{C}_{g} \nabla_{\mathcal{C}} \mathcal{C}_{A}$ is indecomposable, then $\left(g H g^{-1}, \mathcal{C}_{g H} \nabla_{\mathcal{C}_{h}} \mathcal{N}\right)$ is again a type- 1 pair. 
Definition 4.7. Two type-1 pairs $(H, \mathcal{N}),\left(F, \mathcal{N}^{\prime}\right)$ are equivalent if there exists $g \in G$ such that

- $H=g F g^{-1}$, and

- there is an equivalence $\mathcal{N} \simeq \mathcal{C}_{g F} \nabla_{\mathcal{C}_{F}} \mathcal{N}^{\prime}$ of $\mathcal{C}_{H^{-}}$-module categories.

It follows from Lemma $3.5(2)$, that if $(H, \mathcal{N})$ is a type- 1 pair, then $\operatorname{Ind} \mathcal{C}_{\mathcal{C}_{H}}^{\mathcal{N}} \mathcal{N}$ is an exact indecomposable $\mathcal{D}$-module category. We shall see that this establishes a bijective correspondence between equivalence classes of exact indecomposable $\mathcal{D}$-module categories and equivalence classes of type- 1 pairs.

Let's start with an exact $\mathcal{D}$-module category $\mathcal{N}$. It follows from Lemma 3.5 (1) that $\operatorname{Re} s_{\mathcal{C}}^{\mathcal{D}} \mathcal{N}$ is exact. Then, we can decompose it as $\operatorname{Re} s_{\mathcal{C}}^{\mathcal{D}} \mathcal{N}=$ $\oplus_{i=1}^{n} \mathcal{N}_{i}$, into a direct sum of exact indecomposable $\mathcal{C}$-module categories. Denoted by $\overline{\mathcal{N}}_{i}$ the equivalence class of the $\mathcal{C}$-module $\mathcal{N}_{i}$, and by $X_{\mathcal{N}}=$ $\left\{\overline{\mathcal{N}_{i}}: i=1 \ldots n\right\}$.

The group $G$ acts on the set $X_{\mathcal{N}}$. Namely, $g \cdot \overline{\mathcal{N}_{i}}=\overline{\mathcal{N}_{j}}$, if $\mathcal{C}_{g} \nabla_{\mathcal{C}} \mathcal{N}_{i} \simeq \mathcal{N}_{j}$ as $\mathcal{C}$-modules. This action is transitive since $\mathcal{N}$ is indecomposable as a $\mathcal{D}$ module. The next result is well-known.

Lemma 4.8. If $\mathcal{N}, \mathcal{M}$ are equivalent $\mathcal{D}$-module categories, then $X_{\mathcal{M}} \simeq X_{\mathcal{N}}$ as $G$-sets. If $H=\operatorname{Stab}\left(\overline{\mathcal{N}_{1}}\right):=\left\{f \in G \mid f \cdot \overline{\mathcal{N}_{1}}=\overline{\mathcal{N}_{1}}\right\}$, then $X_{\mathcal{N}} \simeq G / H$ as $G$-sets.

Proposition 4.9. [7, Proposition 4.6] Let $(H, \mathcal{N})$ and $(F, \mathcal{M})$ be two type-1 pairs. The following statements are equivalent.

1. There exists an equivalence of $\mathcal{D}$-module categories

$$
\operatorname{Ind}_{\mathcal{C}_{H}}^{\mathcal{D}} \mathcal{N} \simeq \operatorname{Ind}_{\mathcal{C}_{F}}^{\mathcal{D}} \mathcal{M}
$$

2. The type-1 pairs $(H, \mathcal{N}),(F, \mathcal{M})$ are equivalent.

Proof. (1) $\Rightarrow(2)$ If Ind $\mathcal{C}_{\mathcal{C}_{H}}^{\mathcal{D}} \mathcal{N}$, Ind ${ }_{\mathcal{C}_{F}}^{\mathcal{D}} \mathcal{M}$ are equivalent as $\mathcal{C}$-modules, then, by Lemma 4.8(1), $X_{\operatorname{Ind}_{\mathcal{C}_{H} \mathcal{N}}^{\mathcal{D}}} \simeq X_{\operatorname{Ind}_{\mathcal{C}_{F} \mathcal{M}}^{\mathcal{D}}}$ as $G$-sets.

Decompose $\operatorname{Res}_{\mathcal{C}}^{\mathcal{D}}\left(\operatorname{Ind}_{\mathcal{C}_{H}}^{\mathcal{D}} \mathcal{N}\right)$ into a direct sum $\oplus_{i=1}^{n} \mathcal{A}_{i}$ of indecomposable $\mathcal{C}$-module categories. Since the module category $\operatorname{Res}_{C}^{\mathcal{C}_{H}} \mathcal{N}$ is included in $\operatorname{Res}_{\mathcal{C}}^{\mathcal{D}}\left(\operatorname{Ind}_{\mathcal{C}_{H}}^{\mathcal{D}} \mathcal{N}\right)$, and it remains indecomposable as $\mathcal{C}$-module, we can assume that $\mathcal{A}_{1} \simeq \operatorname{Res}_{\mathcal{C}}^{\mathcal{C}_{H}} \mathcal{N}$. It is not difficult to see that $\operatorname{Stab}\left(\overline{\operatorname{Res_{C}\mathcal {C}_{H}\mathcal {N}}}\right)=H$. By Lemma $4.8, G / H \simeq G / F$, thus, there exist $g \in G$ such that $H=g F g^{-1}$.

The restriction of the equivalence (4.4), gives an equivalence of $\mathcal{C}_{H}$-modules

$$
\mathcal{C}_{g F} \otimes_{\mathcal{C}_{F}} \mathcal{M} \simeq \mathcal{C}_{H} \otimes_{\mathcal{C}_{H}} \mathcal{N} \simeq \mathcal{N}
$$

where the $\mathcal{C}_{H}$-action over $\mathcal{C}_{g F}$ is induced by the tensor product of $\mathcal{C}$, and is well defined since $H g=g F$. 
$(2) \Rightarrow(1)$ Let $g \in G$ such that $H=g F g^{-1}$ and $\mathcal{C}_{g F} \bigotimes_{\mathcal{C}_{F}} \mathcal{M} \simeq \mathcal{N}$ as $\mathcal{C}_{H}$-modules. For any $a \in G$, there are equivalences of right $\mathcal{C}_{F}$-modules

$$
\mathcal{C}_{a H} \bigotimes_{\mathcal{C}_{H}} \mathcal{C}_{g F} \simeq\left(\mathcal{C}_{a} \nabla_{\mathcal{C}} \mathcal{C}_{H}\right) \bigotimes_{\mathcal{C}_{H}} \mathcal{C}_{g F} \simeq \mathcal{C}_{a} \nabla_{\mathcal{C}}\left(\mathcal{C}_{H} \otimes_{\mathcal{C}_{H}} \mathcal{C}_{g F}\right) \simeq \mathcal{C}_{a} \bigotimes_{\mathcal{C}} \mathcal{C}_{g F} \simeq \mathcal{C}_{a g F},
$$

where the right $\mathcal{C}_{F}$-module structrure is given by tensor product.

Let $\left\{t_{1}, \ldots, t_{n}\right\}$ be a set of representative of the cosets of $G / F$, thus $\left\{t_{1} g^{-1}, \ldots, t_{n} g^{-1}\right\}$ is a set of representative of the cosets of $G / H$. Using (4.5), we have $\mathcal{D}$-module equivalences

$$
\begin{aligned}
\mathcal{D} \bigotimes_{\mathcal{C}_{F}} \mathcal{M} & \simeq \oplus_{i=1}^{n} \mathcal{C}_{t_{i} F} \bigotimes_{\mathcal{C}_{F}} \mathcal{M} \simeq \oplus_{i=1}^{n}\left(\mathcal{C}_{t_{i} g^{-1} H} \bigotimes_{\mathcal{C}_{H}} \mathcal{C}_{g F}\right) \bigotimes_{\mathcal{C}_{F}} \mathcal{M} \\
& \simeq \oplus_{i=1}^{n} \mathcal{C}_{t_{i} g^{-1} H} \bigotimes_{\mathcal{C}_{H}}\left(\mathcal{C}_{g F} \bigotimes_{\mathcal{C}_{F}} \mathcal{M}\right) \\
& \simeq \oplus_{i=1}^{n} \mathcal{C}_{t_{i} g^{-1} H} \bigotimes_{\mathcal{C}_{H}} \mathcal{N} \simeq \mathcal{D} \bigotimes_{\mathcal{C}_{H}} \mathcal{N}
\end{aligned}
$$

Now, we shall prove that the map

$$
(H, \mathcal{N}) \longmapsto \operatorname{Ind}_{\mathcal{C}_{H}}^{\mathcal{D}} \mathcal{N}
$$

gives a first step to classify exact indecomposable $\mathcal{D}$-module categories. The proof of the following Theorem follows the same steps as the proof of $[10$, Proposition 12] in the semisimple case.

Theorem 4.10. [10, Proposition 12] There exists a bijection between

- equivalence classes of exact indecomposable $\mathcal{D}$-modules, and

- equivalence classes of type-1 pairs $(H, \mathcal{N})$.

Proof. Take a type-1 pair $(H, \mathcal{N})$. Then $\operatorname{Ind}_{\mathcal{C}_{H}}^{\mathcal{D}} \mathcal{N}$ is an exact indecomposable $\mathcal{D}$-module. By Proposition 4.9, the equivalence class of this module category does not depend on the equivalence class of the pair $(H, \mathcal{N})$. The type-1 pair associated to $\operatorname{Ind} \mathcal{C}_{\mathcal{C}_{H}}^{\mathcal{D}} \mathcal{N}$ is $(H, \mathcal{N})$. This follow from the first part of the proof of Proposition 4.9.

Let $\mathcal{M}$ be an exact indecomposable $\mathcal{D}$-module. We shall construct a type-1 pair $(H, \mathcal{N})$ such that $\mathcal{M} \simeq \operatorname{Ind}_{\mathcal{C}_{H}}^{\mathcal{D}} \mathcal{N}$. Let $\operatorname{Res}_{\mathcal{C}}^{\mathcal{D}} \mathcal{M}=\oplus_{i=1}^{n} \mathcal{M}_{i}$ be a decomposition, where $\mathcal{M}_{i}$ is an exact indecomposable $\mathcal{C}$-module. Consider the action of $G$ over the $X_{\mathcal{M}}$ as described before.

Let $H:=\operatorname{Stab}\left(\mathcal{M}_{1}\right)=\left\{f \in G: \mathcal{C}_{f} \otimes_{\mathcal{C}} \mathcal{M}_{1} \simeq \mathcal{M}_{1}\right.$ as $\mathcal{C}$-modules $\}$. Set $\mathcal{N}:=\mathcal{M}_{1}$. The action $\bar{\otimes}: \mathcal{D} \times \mathcal{M} \rightarrow \mathcal{M}$ restricts to an action $\bar{\otimes}: \mathcal{C}_{H} \times \mathcal{N} \rightarrow$ $\mathcal{N}$, inducing a structure of $\mathcal{C}_{H}$-module over $\mathcal{N} . \mathcal{N}$ is an exact $\mathcal{C}_{H}$-module category since $\mathcal{M}$ is an exact $\mathcal{C}$-module. $\mathcal{N}$ is an indecomposable $\mathcal{C}_{H}$-module since $\mathcal{N}$ is indecomposable as a $\mathcal{C}$-module. Hence, we obtain a type-1 pair $(H, \mathcal{N})$. It follows from Lemma 3.4 that $\mathcal{M} \simeq \operatorname{Ind}_{\mathcal{C}_{H}}^{\mathcal{D}} \mathcal{N}$ as $\mathcal{D}$-modules.

4.2. Second step. To any type-1 pair, we shall associate a type-A datum. We shall introduce a new equivalence relation of type-A data, such that there exists a bijection between equivalence classes of type-1 pairs and typeA data. 
Proposition 4.11. Let $A \in \mathcal{C}$ be an algebra such that $\mathcal{C}_{A}$ is an exact indecomposable $\mathcal{C}$-module category, and $H \subseteq G$ be a subgroup. The following statements are equivalent.

1. There exists an exact indecomposable $\mathcal{C}_{H}$-module category $\mathcal{N}$ such that $\operatorname{Res}_{\mathcal{C}}^{\mathcal{C}_{H}} \mathcal{N} \simeq \mathcal{C}_{A}$.

2. There are $\mathcal{C}$-module equivalences $\Psi_{g}: \mathcal{C}_{g} \nabla_{\mathcal{C}} \mathcal{C}_{A} \rightarrow \mathcal{C}_{A}, g \in H$, together with natural $\mathcal{C}$-module transformations

$$
m_{f, g}: \Psi_{f g}\left(M_{f, g} \otimes \operatorname{Id}_{\mathcal{C}_{A}}\right) \rightarrow \Psi_{f}\left(\operatorname{Id}_{\mathcal{C}_{f}} \otimes \Psi_{g}\right)
$$

$f, g \in H$, such that $\Psi_{1}$ is the tensor product, and

$$
\begin{aligned}
& (4.7) \quad\left(m_{f, g}\left(\operatorname{Id}_{\mathcal{C}_{f}} \otimes \operatorname{Id}_{\mathcal{C}_{g}} \nabla \Psi_{h}\right)\right) \circ\left(m_{f g, h}\left(M_{f, g} \otimes \operatorname{Id}_{\mathcal{C}_{h}} \otimes \operatorname{Id}_{\mathcal{C}_{A}}\right)\right) \\
& =\left(\Psi_{f}\left(\operatorname{id}_{\mathcal{C}_{f}} \nabla m_{g, h}\right)\right) \circ\left(m_{f, g h}\left(\operatorname{Id}_{\mathcal{C}_{f}} \nabla M_{g, h} \nabla \operatorname{Id}_{\mathcal{C}_{A}}\right)\right) \circ\left(\Psi_{f g h}\left(\alpha_{f, g, h} \nabla \operatorname{id} \mathcal{C}_{A}\right)\right),
\end{aligned}
$$

for any $f, g, h \in H$.

3. There exists a type- $A$ datum $\left(H,\left\{A_{g}\right\}_{g \in H}, \beta\right)$ for the module $\mathcal{C}_{A}$.

Proof. 1) $\Rightarrow 2$ ). For any $g \in H$, define $\Psi_{g}$ the restriction to $\mathcal{C}_{g}$ of the action of $\mathcal{C}_{H}$ to $\mathcal{C}_{A}$, that is

$$
\Psi_{g}: \mathcal{C}_{g} \otimes_{\mathcal{C}} \mathcal{C}_{A} \rightarrow \mathcal{C}_{A}, \quad \Psi_{g}(X \otimes V)=X \bar{\otimes} V
$$

for any $X \in \mathcal{C}_{g}, V \in \mathcal{C}_{A}$. $\Psi_{g}$ is a $\mathcal{C}$-module functor since the action is associative, and it is an equivalence since $\mathcal{C}_{A}$ is indecomposable. If $m$ denotes the associativity of the $\mathcal{C}_{H}$-module $\mathcal{N}$, then, for any $f, g \in H, X \in \mathcal{C}_{f}, Y \in$ $\mathcal{C}_{g}, V \in \mathcal{N}$ define

$$
\left(m_{f, g}\right)_{X, Y, V}=m_{X, Y, V}
$$

The associativity axiom (2.1) implies (4.7).

$2) \Rightarrow 3)$. Using Lemma $4.1(2)$, there are $\mathcal{C}$-module functors $\Psi_{g}:\left(\mathcal{C}_{g}\right)_{A} \rightarrow$ $\mathcal{C}_{A}$. Here, we are abusing of the notation denoting also by $\Psi_{g}$ the composition of the functors $\Psi_{g}$ with the equivalences $\left(\mathcal{C}_{g}\right)_{A} \simeq \mathcal{C}_{g} \otimes \mathcal{C}_{A}$ of Lemma 4.1 (2). By [5, Prop. 3.11] any module functor between exact module categories is exact, then there exists an object $A_{h} \in{ }_{A}\left(\mathcal{C}_{h}\right)_{A}$ such that

$$
\Psi_{h}=-\otimes_{A} A_{h^{-1}} .
$$

Bimodule $A_{g}$ is invertible since $\Psi_{h}$ is an equivalence, for any $h \in H$. As the functors $\Psi_{f h}\left(M_{f, h} \otimes \operatorname{Id}_{\mathcal{C}_{A}}\right), \Psi_{f}\left(\operatorname{Id}_{\mathcal{C}_{f}} \otimes \Psi_{h}\right)$ are equivalent, then there are bimodule isomorphisms $A_{f} \otimes_{A} A_{h} \simeq A_{f h}$, for any $f, h \in H$.

Since $\Psi_{1}$ is the identity functor, then $A_{1}=A$. As $\mathcal{C}$-module $\operatorname{Res}_{\mathcal{C}}^{\mathcal{C}_{H}} \mathcal{N}$ is indecomposable, then the identity functor $\operatorname{Id}_{\mathcal{N}}$ is a simple object. Arguing as in Lemma 4.1 (3), we obtain that, for any $f, g \in H$ the natural isomorphisms $m_{f, g}$ are determined by scalars $\beta_{f, g} \in \mathbb{k}^{\times}$. Equation (4.7) implies (4.3). Thus, $\left(H,\left\{A_{g}\right\}_{g \in H}, \beta\right)$ is a type-A datum for $\mathcal{C}_{A}$.

$3) \Rightarrow 1$ ). We define an action of $\mathcal{C}_{H}$ on $\mathcal{C}_{A}$ as follows:

$$
X \bar{\otimes} V=(X \otimes V) \otimes_{A} A_{f^{-1}} \in \mathcal{C}_{A},
$$


for any $f \in H, X \in \mathcal{C}_{f}, V \in \mathcal{C}_{A}$. The associativity is defined as follows. For any $f, h \in H, X \in \mathcal{C}_{f}, Y \in \mathcal{C}_{h}, V \in \mathcal{C}_{A}$ define

$$
m_{X, Y, V}=\beta_{f, h}\left(\operatorname{id}_{X \otimes Y \otimes V} \otimes \phi_{f^{-1}, h^{-1}}\right):(X \otimes Y) \bar{\otimes} V \rightarrow X \bar{\otimes}(Y \bar{\otimes} V),
$$

where $\phi_{f, h}: A_{f h} \rightarrow A_{f} \otimes_{A} A_{h}$ are the $A$-bimodule isomorphisms. Here, for simplicity, we are omitting the associativity isomorphisms of $\mathcal{D}$.

In conditions of Proposition 4.11, shall say that $\left(H,\left\{A_{g}\right\}_{g \in H}, \beta\right)$ is the associated type-A datum to the type- 1 pair $(H, \mathcal{N})$. We have to explain now, what happens with the type-A data, if we change the equivalence class of the $\mathcal{C}_{H}$-module category $\mathcal{N}$.

Proposition 4.12. Assume $(H, \mathcal{N})$, and $\left(H, \mathcal{N}^{\prime}\right)$ are two type-1 pairs. As-

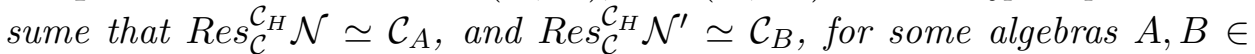
$\mathcal{C}$. Then, the associated type- $A$ data $\left(H,\left\{A_{g}\right\}_{g \in H}, \beta\right),\left(H,\left\{B_{f}\right\}_{f \in H}, \gamma\right)$ are equivalent if and only if $\mathcal{N} \simeq \mathcal{N}^{\prime}$ as $\mathcal{C}_{H}$-module categories.

Proof. Assume that $(\Phi, c): \mathcal{C}_{B} \rightarrow \mathcal{C}_{A}$ is an equivalence of $\mathcal{C}_{H}$-module categories. Then, there exists an object $C \in{ }_{B} \mathcal{C}_{A}$ such that $\Phi=-\otimes_{B} C$. Since $\Phi$ is a $\mathcal{C}_{H}$-module functor, then the existence of natural isomorphisms

$$
c_{X, V}:\left((X \otimes V) \otimes_{B} B_{h^{-1}}\right) \otimes_{B} C \rightarrow\left(X \otimes\left(V \otimes_{B} C\right)\right) \otimes_{A} A_{h^{-1}},
$$

for any $h \in H, X \in \mathcal{C}_{h}$, implies that $B_{h} \otimes_{B} C \simeq C \otimes_{A} A_{h}$, for any $h \in H$ and the isomorphisms $c_{X, V}$ are determined by scalars $\tau_{h}$. Equation (2.3) implies that the type-A data $\left(H,\left\{A_{g}\right\}_{g \in H}, \beta\right),\left(H,\left\{B_{f}\right\}_{f \in F}, \gamma\right)$ are equivalent.

Now, we will show how the type-A datum is modified if we change the module category $\mathcal{N}$ by $\mathcal{C}_{g H} \otimes_{\mathcal{C}_{H}} \mathcal{N}$. For this, we must understand how the associativity isomorphisms $m_{f, h}$, described in (4.6), are modified.

Let be $(H, \mathcal{N})$ a type-1 pair, and $g \in G$. Assume that $\operatorname{Res}_{\mathcal{C}}^{\mathcal{C}_{H}} \mathcal{N} \simeq \mathcal{C}_{A}$, for some algebra $A \in \mathcal{C}$. For any $h \in H$, let $\Psi_{h}: \mathcal{C}_{h} \otimes_{\mathcal{C}} \mathcal{C}_{A} \rightarrow \mathcal{C}_{A}$ be the restrictions of the action of $\mathcal{C}_{H}$ on $\mathcal{N}$. The category $\mathcal{C}_{g H} \otimes_{\mathcal{C}_{H}} \mathcal{N}$ has an action of $\mathcal{C}_{g g^{-1}}$, such that

$$
\operatorname{Res}_{\mathcal{C}}^{\mathcal{C}^{g H g^{-1}}}\left(\mathcal{C}_{g H} \nabla_{\mathcal{C}_{H}} \mathcal{N}\right) \simeq \mathcal{C}_{g} \nabla_{\mathcal{C}} \mathcal{C}_{A}
$$

Let $\widetilde{\Psi}_{h}: \mathcal{C}_{g h g^{-1}} \otimes_{\mathcal{C}} \mathcal{C}_{g} \otimes_{\mathcal{C}} \mathcal{C}_{A} \rightarrow \mathcal{C}_{g} \otimes_{\mathcal{C}} \mathcal{C}_{A}$ be the restrictions of the action of $\mathcal{C}_{g H g^{-1}}$ on the category $\mathcal{C}_{g} \otimes_{\mathcal{C}} \mathcal{C}_{A}$. Explicitly, for any $h \in H$

$$
\widetilde{\Psi}_{h}=\left(\operatorname{Id}_{g} \otimes \Psi_{h}\right)\left(\bar{M}_{g, h}\left(\operatorname{Id}_{g h} \otimes M_{g^{-1}, g}\right)\left(\bar{M}_{g h, g^{-1}} \otimes \operatorname{Id}_{g}\right) \otimes \operatorname{Id}_{\mathcal{M}}\right) .
$$

Here we denote $\mathcal{M}=\mathcal{C}_{A}$, and for any $f \in G, \operatorname{Id}_{f}=\operatorname{Id}_{\mathcal{C}_{f}}$. Recall the definition given in (3.2) of functors $\bar{M}_{g, h}$. For any $f, h \in H$ let

$$
\widetilde{m}_{f, h}: \widetilde{\Psi}_{f h}\left(M_{g f g^{-1}, g h g^{-1}} \otimes \operatorname{Id}_{\mathcal{C}_{g} \bigotimes_{\mathcal{C}} \mathcal{M}}\right) \rightarrow \widetilde{\Psi}_{f}\left(\operatorname{Id}_{\mathcal{C}_{g f g^{-1}}} \otimes \widetilde{\Psi}_{h}\right),
$$

be the natural isomorphisms obtained from the associativity of the module category $\mathcal{C}_{g H} \nabla_{\mathcal{C}_{H}} \mathcal{N}$ as in (4.6). Since $\mathcal{M}$ is an indecomposable $\mathcal{C}$-module category, there exists a 2-cochain $\gamma: g H^{-1} \times g g^{-1} \rightarrow \mathbb{k}^{\times}$such that $\widetilde{m}_{f, h}=\gamma\left(g f g^{-1}, g h g^{-1}\right)$ id, for any $f, h \in H$. 
From now on, for any $f, g \in G$ we shall denote $f^{g}=g f g^{-1}$. If $\eta: G \times G \rightarrow$ $\mathbb{k}^{\times}$is a 2-cochain, we denote $\eta^{g}(f, h)=\eta\left(f^{g}, h^{g}\right)$.

Lemma 4.13. Under the above assumptions, there exists a 2-cochain $\Omega_{g}^{A}$ : $G \times G \rightarrow \mathbb{k}^{\times}$, that depends on the Morita class of $A$, such that

$$
\gamma^{g} \Omega_{g}^{A}=\beta \text {. }
$$

Proof. We are going to abuse of the notation and use the monoidal equivalence

$$
\operatorname{Fun}_{\mathcal{C}}\left(\left(\mathcal{C}_{f}\right)_{A},\left(\mathcal{C}_{g}\right)_{A}\right) \simeq{ }_{A}\left(\mathcal{C}_{f^{-1} g}\right)_{A}^{\text {op }}
$$

So that composition of functors in the first category correspond to the monoidal product in the second category. Note that, our assumption that $\mathcal{D}$ is skeletal implies that ${ }_{A}\left(\mathcal{C}_{f^{-1} g}\right)_{A}^{\text {op }}$ is skeletal.

If $f, h \in H$, then, using (4.8), we get that the functor $\widetilde{\Psi}_{f h}\left(M_{f^{g}, h^{g}} \otimes \operatorname{Id}_{\mathcal{M}}\right)$ equals

$$
\begin{aligned}
& =\left(\operatorname{Id}_{g} \otimes \Psi_{f h}\right)\left(\bar{M}_{g, f h}\left(\operatorname{Id}_{g f h} \otimes M_{g^{-1}, g}\right)\left(\bar{M}_{g f h, g^{-1}} \otimes \operatorname{Id}_{g}\right) M_{f^{g}, h^{g}} \otimes \operatorname{Id}_{\mathcal{M}}\right) \\
& =\beta^{-1}(f, h)\left(\left(\operatorname{Id}_{g} \otimes \Psi_{f}\right)\left(\operatorname{Id}_{g} \otimes \operatorname{Id}_{f} \otimes \Psi_{h}\right)\left(\operatorname{Id}_{g} \otimes \bar{M}_{f, h}\right)\right) \\
& \left(\bar{M}_{g, f h}\left(\operatorname{Id}_{g f h} \otimes M_{g^{-1}, g}\right)\left(\bar{M}_{g f h, g^{-1}} \otimes \operatorname{Id}_{g}\right) M_{f^{g}, h^{g}} \otimes \operatorname{Id}_{\mathcal{M}}\right) .
\end{aligned}
$$

Here, the second equality follows from Proposition 4.11, where the natural isomorphisms $m_{f, h}$ are equal to $\beta(f, h)$ id, hence

$$
\beta(f, h) \Psi_{f g}\left(M_{f, g} \otimes \operatorname{Id}_{\mathcal{C}_{A}}\right)=\Psi_{f}\left(\operatorname{Id}_{\mathcal{C}_{f}} \otimes \Psi_{g}\right) .
$$

On the other hand, the functor $\widetilde{\Psi}_{f}\left(\operatorname{Id}_{f g} \otimes \widetilde{\Psi}_{h}\right)$ equals

$$
\begin{aligned}
& =\left(\operatorname{Id}_{g} \otimes \Psi_{f}\right)\left(\bar{M}_{g, f}\left(\operatorname{Id}_{g f} \otimes M_{g^{-1}, g}\right)\left(\bar{M}_{g f, g^{-1}} \otimes \operatorname{Id}_{g}\right)\left(\operatorname{Id}_{f^{g}} \otimes \operatorname{Id}_{g} \otimes \Psi_{h}\right)\right. \\
& \left.\left(\operatorname{Id}_{f^{g}} \otimes \bar{M}_{g, h}\right)\left(\operatorname{Id}_{f^{g}} \otimes \operatorname{Id}_{g h} \otimes M_{g, g^{-1}}\right)\left(\operatorname{Id}_{f^{g}} \otimes \bar{M}_{g h, g^{-1}}\right) \otimes \operatorname{Id}_{\mathcal{M}}\right) \\
& =\left(\operatorname{Id}_{g} \otimes \Psi_{f}\right)\left(\left(\operatorname{Id}_{g} \otimes \operatorname{Id}_{f} \otimes \Psi_{h}\right)\left(\bar{M}_{g, f} \otimes \operatorname{Id}_{h}\right)\left(\operatorname{Id}_{g f} \otimes M_{g^{-1}, g} \otimes \operatorname{Id}_{h}\right)\right. \\
& \left(\bar{M}_{g f, g^{-1}} \otimes \operatorname{Id}_{g} \otimes \operatorname{Id}_{h}\right)\left(\operatorname{Id}_{f^{g}} \otimes \bar{M}_{g, h}\right)\left(\operatorname{Id}_{f^{g}} \otimes \operatorname{Id}_{g h} \otimes M_{g^{-1}, g}\right) \\
& \left.\left(\operatorname{Id}_{f^{g}} \otimes \bar{M}_{g h, g^{-1}}\right) \otimes \operatorname{Id}_{\mathcal{M}}\right) .
\end{aligned}
$$

Since for any $f, h \in H$, we have that

$$
\widetilde{\Psi}_{f}\left(\operatorname{Id}_{f^{g}} \otimes \widetilde{\Psi}_{h}\right)=\gamma\left(f^{g}, h^{g}\right) \widetilde{\Psi}_{f h}\left(M_{f^{g}, h^{g}} \otimes \operatorname{Id}_{\mathcal{M}}\right),
$$

we deduce that

$$
\begin{aligned}
& \left(M_{f^{g}, h^{g}} \otimes \operatorname{Id}_{g}\right)\left(\operatorname{Id}_{f^{g}} \otimes M_{g h, g^{-1}} \otimes \operatorname{Id}_{g}\right)\left(\operatorname{Id}_{f^{g}} \otimes M_{g, h} \otimes \operatorname{Id}_{g^{-1}} \otimes \operatorname{Id}_{g}\right) \\
& \left(M_{g f, g^{-1}} \otimes \operatorname{Id}_{g} \otimes \operatorname{Id}_{h} \otimes \operatorname{Id}_{g^{-1}} \otimes \operatorname{Id}_{g}\right)\left(M_{g, f} \otimes \operatorname{Id}_{\mathcal{C}_{g^{-1}} \otimes \mathcal{C}_{g} \otimes \mathcal{C}_{h} \otimes \mathcal{C}_{g^{-1}} \otimes \mathcal{C}_{g}}\right) \\
& \left(\operatorname{Id}_{g} \otimes \operatorname{Id}_{f} \otimes \bar{M}_{g^{-1}, g} \otimes \operatorname{Id}_{\mathcal{C}_{h} \bowtie \mathcal{C}_{g^{-1}} \otimes \mathcal{C}_{g}}\right)\left(\operatorname{Id}_{g} \otimes \bar{M}_{f, h} \otimes \operatorname{Id}_{\mathcal{C}_{g^{-1}} \otimes \mathcal{C}_{g}}\right) \\
& \left(\bar{M}_{g, f h} \otimes \operatorname{Id}_{\mathcal{C}_{g^{-1}} \otimes \mathcal{C}_{g}}\right)\left(\bar{M}_{g f h, g^{-1}} \otimes \operatorname{Id}_{g}\right) \otimes \operatorname{Id}_{\mathcal{M}}=\frac{\beta(f, h)}{\gamma\left(f^{g}, h^{g}\right)} \operatorname{Id}_{\mathcal{C}_{g f h g^{-1}} \otimes \mathcal{C}_{g} \bowtie \mathcal{M}} .
\end{aligned}
$$


The left hand side of equality (4.10) is equal to a scalar multiple of the identity. We denote this scalar by $\Omega_{g}^{A}$, hence we obtain the desired result.

Remark 4.14. If $G=C_{2}$ is the cyclic group of 2 elements, one can verify that the functor on the left part of (4.10) equals the identity, thus $\Omega_{g}^{A}=1$.

Definition 4.15. Two type-A data $\left(H,\left\{A_{h}\right\}_{h \in H}, \beta\right),\left(F,\left\{B_{f}\right\}_{f \in F}, \gamma\right)$ are $G$-equivalent if there exists $g \in G$, and an invertible $(B, A)$-bimodule $C \in$ ${ }_{B}\left(\mathcal{C}_{g}\right)_{A}$ such that

- $F=g g^{-1}$

- $B_{g h g^{-1}} \otimes_{B} C \simeq C \otimes_{A} A_{h}$, for all $h \in H$;

- the class of $\beta^{-1} \Omega_{g}^{A} \gamma^{g}$ is trivial in $H^{2}\left(H, \mathbb{k}^{\times}\right)$.

Remark 4.16. If $\left(H,\left\{A_{g}\right\}_{g \in H}, \beta\right)$ and $\left(H,\left\{B_{f}\right\}_{f \in F}, \gamma\right)$ are $G$-equivalent, then $\mathcal{C}_{A}, \mathcal{C}_{B}$ are equivalent $\mathcal{C}$-module categories.

Combining Theorem 4.10, Proposition 4.11 and Lemma 4.13 we get the next result.

Theorem 4.17. There exists a bijection between

- equivalence classes of exact indecomposable $\mathcal{D}$-modules,

- $G$-equivalence classes type-A datum, and

- equivalence classes of type-1 pairs.

4.3. Pointed fusion categories. Let us show that our results, when applied to a pointed fusion category, agree with the results obtained in [11].

Assume $G$ is a finite group, and $\omega \in H^{3}\left(G, \mathbb{k}^{\times}\right)$is a 3-cocycle. If $\mathcal{D}=$ $\mathcal{C}(G, \omega)$, then $\mathcal{D}$ is a $G$-extension of the category vect $\mathbb{k}_{\mathbb{k}}$ of finite-dimensional vector spaces.

Theorem 4.17 implies, in this case, that exact indecomposable $\mathcal{D}$-module categories are parametrized by pairs $(H, \beta)$, where $H \subseteq G$ is a group, and $\beta \in C^{2}\left(H, \mathbb{k}^{\times}\right)$is a 2 -cochain such that $d \beta \omega=1$. This parametrization coincides with the one given in [5].

For such pair $(H, \beta)$, denote $\mathcal{M}_{0}(H, \beta)$ the associated exact indecomposable $\mathcal{D}$-module category. As abelian categories $\mathcal{M}_{0}(H, \beta)=\mathcal{C}(G, \omega)_{\mathbb{k}_{\beta} H}$.

The equivalence classes of such module categories agrees with the one described in [11]. In [11, Thm 1.1], the author proves the following result.

Theorem 4.18. Assume $L, H \subseteq G$ are two groups, and $\beta \in C^{2}\left(H, \mathbb{k}^{\times}\right)$, $\xi \in C^{2}\left(L, \mathbb{k}^{\times}\right)$are 2-cochains such that $d \beta=\omega^{-1}=d \xi$. There exists an equivalence of module categories between $\mathcal{M}_{0}(H, \beta), \mathcal{M}_{0}(L, \xi)$ if and only if there exists $g \in G$ such that $H=g L g^{-1}$, and the class of $\left.\xi^{-1} \beta^{g} \Omega_{g}\right|_{L \times L}$ is trivial in $H^{2}\left(L, \mathbb{k}^{\times}\right)$.

In the above theorem $\Omega_{g}: G \times G \rightarrow \mathbb{k}$ is defined by

$$
\Omega_{g}(f, h)=\frac{\omega\left(f^{g}, g, h\right)}{\omega\left(f^{g}, h^{g}, g\right) \omega(g, f, h)},
$$


for any $f, h \in G$. Note that there is a difference between this definition of $\Omega_{g}$ and the one presented in [11]. The difference comes from the fact that in loc. cit the author uses $\omega^{-1}$ instead of $\omega$.

To prove that our classification agrees with the one in [11], we shall prove that the 2-cochain $\Omega_{g}^{\mathbb{k}}$ is cohomologous to $\Omega_{g}$, for any $g \in G$. Here $\mathbb{k}$ is the algebra representing the unique (equivalence class) exact indecomposable module category over vect $\mathbb{k}$. To this end, lets compute explicitly $\Omega_{g}^{\mathbb{k}}$. Calculating the functor of the left hand side of (4.10), we get that

$$
\Omega_{g}^{\mathbb{k}}(f, h)=\frac{\omega\left(f^{g}, g, h\right) \omega\left(f^{g}, g h, g^{-1}\right)}{\omega(g, f, h) \omega\left(g f, g^{-1}, g\right)},
$$

for any $f, h \in G$. Using the 3-cocycle condition, we obtain that

$$
\omega\left(f^{g}, g h, g^{-1}\right) \omega\left(f^{g}, h^{g}, g\right) \omega\left(g h, g^{-1}, g\right)=\omega\left(g f h, g^{-1}, g\right) .
$$

Hence

$$
\begin{aligned}
\Omega_{g}^{\mathbb{k}}(f, h) & =\frac{\omega\left(f^{g}, g, h\right) \omega\left(g f h, g^{-1}, g\right)}{\omega(g, f, h) \omega\left(g f, g^{-1}, g\right) \omega\left(f^{g}, h^{g}, g\right) \omega\left(g h, g^{-1}, g\right)} \\
& =\Omega_{g}(f, h) \frac{\omega\left(g f h, g^{-1}, g\right)}{\omega\left(g f, g^{-1}, g\right) \omega\left(g h, g^{-1}, g\right)} .
\end{aligned}
$$

This implies that $\Omega_{g}$ and $\Omega_{g}^{\mathbb{k}}$ are cohomologous.

Remark 4.19. Assume $\mathcal{D}=\mathcal{T} \mathcal{Y}(A, \chi, \tau)$ is a Tambara-Yamagami tensor category, see [12] for its definition. It follows easily that the functor of the left hand side of (4.10) is the identity. Thus, the equivalence classes of indecomposable exact module categories over $\mathcal{T Y}(A, \chi, \tau)$ obtained in [10] remains the same as our case.

4.4. On the results obtained by Meir-Musicantov. In [10] the authors classify indecomposable semisimple module categories over a $G$-graded fusion category, in terms of certain cohomological data. In [10, Section 8] it is explained how to obtain from a type-A datum these cohomological data. We shall briefly recall this correspondence.

Let $\mathcal{M}$ be an exact indecomposable $H$-equivariant $\mathcal{C}$-module category. This means that, for any $h \in H$, we have $\mathcal{C}$-module equivalences

$$
\psi_{h}: \mathcal{C}_{h} \otimes_{\mathcal{C}} \mathcal{M} \rightarrow \mathcal{M}
$$

Since $\mathcal{M}$ is exact, there exists an algebra $A \in \mathcal{C}$, such that $\mathcal{M} \simeq \mathcal{C}_{A}$ as module categories. Also, exactness of $\mathcal{M}$ implies that functors $\psi_{h}$ are exact. Using the equivalence $\mathcal{C}_{h} \nabla_{\mathcal{C}} \mathcal{C}_{A} \simeq\left(\mathcal{C}_{h}\right)_{A}$, the functors $\psi_{h}:\left(\mathcal{C}_{h}\right)_{A} \rightarrow \mathcal{C}_{A}$ are given by $\psi_{h}=-\otimes_{A} D_{h^{-1}}$, where, for any $h \in H, D_{h} \in{ }_{A}\left(\mathcal{C}_{h}\right)_{A}$ is an invertible $A$-bimodule.

Let $\Gamma$ be the group of isomorphism classes of invertible $A$-bimodules in $\mathcal{C}$, and let $\Lambda$ be the group of isomorphism classes of invertible $A$-bimodules in $\mathcal{C}_{H}$. Any invertible invertible $A$-bimodules in $\mathcal{C}_{H}$ must be supported in a 
unique category $\mathcal{C}_{h}$, for some $h \in H$. Thus, we have an exact sequence of groups

$$
1 \rightarrow \Gamma \stackrel{\iota}{\rightarrow} \Lambda \stackrel{\pi}{\rightarrow} H \rightarrow 1
$$

Here, $\pi$ is the map assigning an invertible bimodule the element $h$ where it is supported, and $\iota$ is the inclusion. Surjectivity of $\pi$ follows from the existence of the bimodules $D_{h}$.

Remark 4.20. The group $\Gamma$ is isomorphic to the group of equivalence classes of $\mathcal{C}$-module autoequivalences of $\mathcal{C}_{A}$, and the group $\Lambda$ is isomorphic to the group of equivalence classes of $\mathcal{C}$-module autoequivalences of $\left(\mathcal{C}_{H}\right)_{A}$.

Associated to $\mathcal{M}$ there is a group morphism $\rho: H \rightarrow$ Out $(\Gamma)$, given by

$$
\rho(h)(a)=D_{h} a D_{h}^{-1}
$$

for any $a \in \Gamma$. Define also $Y: H \times H \rightarrow \Gamma$,

$$
Y_{h, f}=D_{h f} D_{f}^{-1} D_{h}^{-1} \text {. }
$$

Denote by $p: \operatorname{Aut}(\Gamma) \rightarrow$ Out $(\Gamma)$ the canonical projection. If there is a lifting $\Phi: H \rightarrow$ Aut $(\Gamma)$ of $\rho$, that is, a morphism that satisfies $\rho=p \Phi$, then the element $Y_{h, f}$ belongs to the center of $\Gamma$ and it is a 2-cocycle with action given by $\Phi$.

Definition 4.21. A $M M$-datum is a collection $(\mathcal{M}, H, \Phi, v, \beta)$, where

- $H \subseteq G$ is a subgroup;

- $\mathcal{M}$ is an exact indecomposable $H$-equivariant $\mathcal{C}$-module category;

- $\Phi: H \rightarrow \operatorname{Aut}(\Gamma)$ is a lifting of $\rho$;

- $v: H \rightarrow Z(\Gamma)$ is a 1-cochain such that $d v=Y$;

- $\beta: H \times H \rightarrow \mathbb{k}^{\times}$is a 2-cochain such that $\omega^{\mathcal{M}} d \beta=1$.

The existence of the pair $(\Phi, v)$ is equivalent to the fact that the exact sequence (4.11) splits. If $\left(H,\left\{A_{g}\right\}_{g \in H}, \beta\right)$ is a type-A datum for the module category $\mathcal{M} \simeq \mathcal{C}_{A}$, then there is a splitting of (4.11) given by

$$
s: H \rightarrow \Lambda, \quad s(h)=A_{h}, \text { for any } h \in H .
$$

In [10, Thm. 2] the authors classify semisimple indecomposable module categories over a fusion $G$-graded category in terms of MM-data.

\section{REFERENCES}

[1] A. Davydov and. D. Nikshych, The Picard crossed module of a braided tensor category, Algebra and Number Theory 7 no. 6 (2013), 1365-1403.

[2] P. Etingof and S. Gelaki, Exact sequences of tensor categories with respect to a module category, Adv. math. 308 (2017), 1187-1208.

[3] P. Etingof, S. Gelaki, D. Nikshych and V. Ostrik, Tensor categories, Lectures notes (2009) 80-83. http://www-math.mit.edu/ etingof/tenscat1.pdf

[4] P. Etingof, D. Nikshych and V. Ostrik, Fusion categories and homotopy theory, Quantum Topol. 1 no. 3 (2010), 209-273.

[5] P. Etingof and V. Ostrik, Finite tensor categories, Mosc. Math. J. 4 no. 3 (2004) , 627-654. 
[6] C. Galindo, Clifford theory for tensor categories, J. London Math. Soc. 83 no. 1 (2011), 57-78.

[7] C. Galindo, Clifford theory for graded fusion categories, Israel J. Math. 192 no. 2 (2012), 841-867.

[8] C.L. Douglas, C. Schommer-Pries and N. Snyder, The balanced tensor product of module categories, preprint arXiv:1406.4204.

[9] J. Greenough. Monoidal 2-structure of Bimodules, J. Algebra 324 (2010), 1818-1859.

[10] E. Meir and E. Musicantov, modules over graded fusion categories, J. Pure Appl. Algebra 216 (2012), 2449-2466.

[11] S. NATALE, On the equivalence of module categories over a group-theoretical fusion category, SIGMA, Symmetry Integrability Geom. Methods Appl. 13 Paper 042, 9 p. (2017).

[12] D. Tambara, S. Yamagami, Tensor categories with fusion rules of self-duality for finite abelian groups, J. Algebra 209 no. 2 (1998), 692-707.

E-mail address: sighana25@gmail.com

URL: https://sites.google.com/site/adrianamejia1209

E-mail address: martin10090@gmail.com, mombelli@mate.uncor.edu

URL: http://www. mate. uncor.edu/ mombelli/welcome.html 